\title{
ORIGINAL RESEARCH \\ Thalamic Damage Predicts the Evolution of Primary-Progressive Multiple Sclerosis at 5 Years
}

S. Mesaros

M.A. Rocca

E. Pagani

M.P. Sormani

M. Petrolini

G. Comi

M. Filippi
BACKGROUND AND PURPOSE: Reliable markers to monitor PPMS are still needed. We investigated whether conventional and DTI measures of thalamic damage are predictive of long-term disability accumulation in PPMS.

MATERIALS AND METHODS: Brain conventional and DTI scans were obtained at baseline and after a mean follow-up of 15 months in 54 patients with PPMS and 8 healthy controls. Patients were reassessed clinically after 5 years. At baseline and follow-up, measures of lesion load, brain atrophy, and NTV were obtained. MD and FA histograms of the NAWM, the whole GM without the thalami, and the thalami were obtained. A multivariate analysis evaluated the predictors of long-term neurologic deterioration.

RESULTS: At follow-up, 35 patients showed disability worsening. At baseline, compared with healthy controls, patients with PPMS had lower NTV $(P<.001)$ and thalamic FA $(P=.002)$ and higher thalamic $(P=.002)$ and whole GM without the thalami $(P=.005) \mathrm{MD}$. During follow-up, the change of thalamic FA was higher in PPMS versus healthy controls $(P=.01)$. Baseline NTV and thalamic DTI quantities differed significantly between patients with PPMS with and without thalamic lesions. Baseline thalamic quantities were significantly correlated with the extent of brain T2 lesions and the severity of NAWM damage. The multivariate model included average NAWM MD (OR $=1.46, P=.005)$ and FA thalamic change $(\mathrm{OR}=0.84, P=.02$ ) as independent predictors of EDSS score deterioration (Nagelkerke $R^{2}=0.55$ ).

CONCLUSIONS: Short-term accrual of thalamic damage and the severity of NAWM involvement predict the long-term accumulation of disability in PPMS.

ABBREVIATIONS: $\mathrm{Cl}=$ confidence interval; DTI = diffusion tensor imaging; $\mathrm{EDSS}=$ Expanded Disability Status Scale; FA = fractional anisotropy; GM = gray matter; LV = lesion volume; $\mathrm{MD}=$ mean diffusivity; $\mathrm{MS}=$ multiple sclerosis; $\mathrm{n} . \mathrm{a}$. = not applicable; NAWM = normal-appearing white matter; n.s. = not significant; NTV = normalized thalamic volume; OR = odds ratio; PD = proton density; PGSE = pulsed-gradient spin-echo echo-planar; PPMS = primary-progressive MS; SIENAX = structural image evaluation, with normalization of atrophy; SPM = statistical parametric mapping; $\mathrm{WM}=$ white matter
$\mathbf{T}$ he mechanisms underlying the steady progression of disability typically seen in patients with PPMS are still poorly understood. ${ }^{1}$ In most of these patients, a discrepancy between conventional MR imaging parameters and clinical disability has been observed. ${ }^{2}$ As a consequence, there has been a great effort in the past few years to image diffuse brain and spinal cord abnormalities by using quantitative MR imaging techniques, which allow obtaining a more complete picture of tissue damage in patients with MS than conventional MR imaging. ${ }^{3-8}$

Several studies have emphasized the role of GM abnormalities and their relationship to clinical disability in PPMS. ${ }^{5-7,9}$ However, only a few studies identified MR imaging markers of GM damage with the potential to predict subsequent disability accumulation. ${ }^{10-12}$ In addition, most of these studies assessed whole GM damage, by using either a histogram-based or a voxelwise approach. Thus, the role of individual GM compartments

\section{Received September 8, 2010; accepted after revision October 18}

From the Neuroimaging Research Unit (S.M., M.A.R., E.P., M.P.S., M.P., M.F.), Division of Neuroscience, Institute of Experimental Neurology, and Department of Neurology (M.A.R., G.C., M.F.), Scientific Institute and University Ospedale San Raffaele, Milan, Italy; and Unit of Biostatistics, Department of Health Services (M.P.S.), University of Genoa, Genoa, Italy.

Please address correspondence to Massimo Filippi, MD, Neuroimaging Research Unit Institute of Experimental Neurology, Scientific Institute and University Hospital San Raffaele, Via Olgettina, 60, 20132 Milan, Italy; e-mail: m.filippi@hsr.it

DOI 10.3174/ajnr.A2430 (eg, cortical and deep GM) in predicting disability worsening remains to be established. Among brain GM structures, several pathologic $^{13,14}$ and imaging ${ }^{15-19}$ studies have demonstrated consistently that thalamic involvement is, from the earliest stages of the disease, a prominent feature of MS. ${ }^{20-23}$

In a longitudinal DTI study of a relatively large cohort of patients with PPMS with a clinical follow-up of approximately 5 years, ${ }^{11}$ we found that baseline GM average MD of the whole brain and baseline EDSS scores ${ }^{24}$ were independent predictors of subsequent clinical deterioration during the study period. Against this background, the objective of this study was to determine the relative contribution of baseline damage to the thalamus (in terms of focal lesions, atrophy, and DTI abnormalities) as well as its change during a 15-month period in predicting the accumulation of disability during the subsequent 5 years in this cohort of patients.

\section{Materials and Methods}

\section{Patients}

As previously reported, ${ }^{11} 54$ patients with a diagnosis of $\mathrm{PPMS}^{1}(27$ women; mean age $=51.3$ years, range $=25-68$ years; median disease duration $=10$ years, range $=2-26$ years) were enrolled. All patients are participating in a long-term prospective follow-up study. Eight age- and sex-matched healthy volunteers with no history of neuro- 
logic disease and with normal results on neurologic examinations served as controls.

Neurologic assessment and EDSS ${ }^{24}$ rating were performed when MR images were obtained and at preplanned time points by the same observer. ${ }^{11}$ In the present study, the median length of the clinical follow-up was 56 months (range $=35-63$ months). At follow-up evaluation, patients were considered clinically worse if they had an EDSS score increase of $\geq 1.0$, when the baseline EDSS score was $<6.0$, or an EDSS score increase of $\geq 0.5$, when baseline EDSS score was $\geq 6.0$. Median EDSS scores were 5.5 (range $=2.5-6.5$ ) at baseline and 6.5 (range $=0.0-9.0)$ at follow-up. At final assessment, 35 patients (65\%) were considered clinically worse.

A comprehensive description of study design, inclusion/exclusion criteria, and demographic and clinical characteristics of the subjects of the study are provided elsewhere. ${ }^{11}$

Local ethics committee approval and written informed consent from all subjects were obtained before study initiation.

\section{Image Acquisition}

Using a $1.5 \mathrm{~T}$ magnet, we acquired the following scans of the brain at baseline and after a median period of 15 months (range $=12-23$ months): 1) dual-echo turbo spin-echo; 2) T1-weighted conventional spin-echo; and 3) PGSE with diffusion gradients applied in 8 noncollinear directions, chosen to cover 3D space uniformly. Twenty-four contiguous axial sections with 5-mm thickness were obtained for dual-echo and T1-weighted scans. For PGSE scans, 10 axial sections with 5-mm thickness were acquired, with the same orientation as the other scans and the second-to-last caudal section positioned to match exactly the central sections of these sets. At follow-up, patients were repositioned following published guidelines. ${ }^{25}$ The complete imageacquisition scheme is detailed elsewhere. ${ }^{6}$

\section{Image Review and Analysis}

Following the identification of T2 and T1 hypointense MS lesions, total LV was measured as previously described. ${ }^{26}$ Thalamic T2 hyperintense lesions were identified.

Using T1-weighted images, we estimated baseline normalized brain volume by using SIENAX ${ }^{27}$ software (www.fmrib.ox.ac.uk/fsl/ siena) and the percentage of brain volume change by using its longitudinal version. ${ }^{28}$

Following the automated segmentation of the brain GM, WM, and CSF from PD- and T2-weighted images, the resulting masks for each tissue type were produced, as previously described. ${ }^{11} \mathrm{MD}$ and FA maps were produced from the PGSE images and coregistered with the dual-echo images. DTI analysis of lesions, as well as of the whole GM and NAWM, has been described elsewhere. ${ }^{11}$

To calculate thalamic volume, we normalized PD-weighted images to the standard Montreal Neurologic Institute space by using the nonlinear deformation implemented in SPM2 (Wellcome Department of Imaging Neuroscience, London, United Kingdom). Then, maps of the left and right thalamus, obtained from the WFU_Pick Atlas (http://www.fmri.wfubmc.edu/software/PickAtlas/), were used as a reference for the manual editing of thalamic regions of interest, which were contoured by using the tool implemented in Jim, Version 5 (www.xinapse.com/manual/index) and transferred to each normalized PD-weighted image. After we revised the template regions of interest, new masks were produced. Thalamic masks were transformed back to the single-subject space after inversion of the transformation (SPM2, deformation toolbox, "invert deformation"), and thalamic volumes were calculated. In each individual, thalamic vol- umes were normalized by using the scaling factor calculated with SIENAX, thus obtaining an NTV. The reproducibility of such a strategy has been assessed previously and resulted in an intraclass correlation coefficient of $0.911 .^{29}$

For the present analysis, average MD and FA values were quantified for the whole GM without the thalami and for the thalami in isolation. To this end, we applied thalamic masks to GM masks both inclusively and exclusively to obtain thalamic GM and GM without the thalami.

\section{Statistical Analysis}

Within-group changes of thalamic and GM without the thalami MR imaging-derived variables between baseline and 15-month follow-up were compared by using a Student $t$ test for paired data. To compare the longitudinal changes of MR imaging-derived variables between patients with PPMS and controls as well as between patients with and those without thalamic lesions, we used a Student $t$ test for nonpaired data.

A univariate logistic regression model adjusted for follow-up duration was used to investigate the role of all the considered MR imaging-derived variables (baseline number of thalamic lesions, change in thalamic lesions, baseline NTV, percentage NTV change, baseline $\mathrm{MD}$ and FA values of GM without the thalami, percentage MD and FA changes of GM without the thalami, baseline thalamic MD and FA values, and percentage thalamic MD and FA changes) as predictors of the probability of having an EDSS deterioration at follow-up. We also ran a multivariate model, including variables found to be significant at the univariate analyses of the previous (baseline EDSS, T2 LV, average lesion MD, average NAWM MD and FA, and number of new $\mathrm{T} 2$ and $\mathrm{T} 1$ lesions at follow-up) $)^{11}$ and present studies. The multivariate analysis was also adjusted for the actual follow-up duration. Univariate correlations between thalamic and other clinical and MR imaging quantities were assessed by using the Spearman rank correlation coefficient.

\section{Results}

Conventional and DTI measures of the NAWM and GM at baseline and 15-month follow-up have been reported previously. ${ }^{11}$ At baseline, T2 visible lesions in the thalami were identified in 29 patients with PPMS (median number of lesions 1 , range $=1-6$ ). During the follow-up, new T2 visible lesions in the thalami were identified in 9 patients with PPMS (7 of them did not have thalamic lesions at baseline).

In Table 1, thalamic volumetry and DTI data as well as DTI data of the GM without the thalami from healthy controls and patients with PPMS at study entry and 15-month follow-up are presented. In healthy controls, NTV and DTI quantities from the thalami and the GM without the thalami remained stable during the 15-month follow-up, whereas during the same study period, patients with PPMS showed a significant worsening of all the MR imaging quantities analyzed, except for average GM without thalami MD.

At baseline, compared with healthy controls, patients with PPMS had lower NTV $(P<.001)$ and thalamic FA $(P=.002)$, as well as higher thalamic MD $(P=.002)$ and MD of the GM without the thalami $(P=.005)$. During follow-up, change of thalamic FA was higher in patients with PPMS versus healthy controls $(P=.01)$. Baseline NTV and thalamic DTI quantities were significantly different between patients with PPMS with and without thalamic T2 visible lesions (Table 2). 


\begin{tabular}{|c|c|c|c|}
\hline Variables & Healthy Controls & $\begin{array}{l}\text { Patients with } \\
\text { PPMS }\end{array}$ & $\begin{array}{c}P \\
\text { Value }\end{array}$ \\
\hline \multicolumn{4}{|l|}{ NTV } \\
\hline Baseline & $6.11(0.48)$ & $4.11(0.95)$ & $<0.001$ \\
\hline$\%$ Change & $-1.9(3.1)$ & $-4.7(6.2)$ & 0.39 \\
\hline$P^{\mathrm{a}}$ & n.s. & $<.0001$ & \\
\hline \multicolumn{4}{|l|}{ Thalamic MD } \\
\hline Baseline & $0.87(0.04)$ & $1.08(0.16)$ & .002 \\
\hline$\%$ Change & $+4.0(8.7)$ & $+2.0(10.2)$ & .61 \\
\hline$P^{\mathrm{b}}$ & n.s. & $<.0001$ & \\
\hline \multicolumn{4}{|l|}{ Thalamic FA } \\
\hline Baseline & $0.23(0.01)$ & $0.21(0.02)$ & .002 \\
\hline$\%$ Change & $-5.0(7.1)$ & $-12.2(7.1)$ & .01 \\
\hline$P^{\mathrm{b}}$ & n.s. & $<.0001$ & \\
\hline \multicolumn{4}{|c|}{ GM without thalami MD } \\
\hline Baseline & $1.02(0.04)$ & $1.08(0.06)$ & .005 \\
\hline$\%$ Change & $+1.0(2.4)$ & $+2.0(6.2)$ & n.s \\
\hline$P^{\mathrm{b}}$ & n.s. & n.s. & \\
\hline \multicolumn{4}{|c|}{ GM without thalami FA } \\
\hline Baseline & $0.15(0.06)$ & $0.14(0.11)$ & n.s. \\
\hline$\%$ Change & $-6.1(12.0)$ & $-16.1(16.1)$ & .06 \\
\hline$P^{\mathrm{b}}$ & n.s. & .009 & \\
\hline
\end{tabular}

${ }^{a}$ Values are means \pm SDs in parentheses.

b Pair-wise comparisons versus baseline values (see the text for statistical analysis).

\begin{tabular}{|c|c|c|c|}
\hline Variables & $\begin{array}{l}\text { PPMS without } \\
\text { Lesions }\end{array}$ & $\begin{array}{l}\text { PPMS with } \\
\text { Lesions }\end{array}$ & $\begin{array}{c}P \\
\text { Value }\end{array}$ \\
\hline \multicolumn{4}{|l|}{ NTV } \\
\hline Baseline & $4.49(0.88)$ & $3.62(0.74)$ & .001 \\
\hline$\%$ Change & $-3.7(5.5)$ & $-5.8(6.5)$ & .22 \\
\hline \multicolumn{4}{|l|}{ Thalamic MD } \\
\hline Baseline & $1.01(0.13)$ & $1.19(0.19)$ & .001 \\
\hline$\%$ Change & $+2.4(10.8)$ & $+0.9(9.1)$ & .60 \\
\hline \multicolumn{4}{|l|}{ Thalamic FA } \\
\hline Baseline & $0.21(0.02)$ & $0.20(0.02)$ & .05 \\
\hline$\%$ Change & $-12.8(7.1)$ & $-11.7(7.2)$ & .60 \\
\hline
\end{tabular}

a Values are means \pm SDs in parentheses. See the text for statistical analysis.

At baseline, in patients with PPMS, significant correlations were found between the following: NTV versus the number of thalamic T2 visible lesions $(r=-0.46, P=.001)$, T2 LV $(r=$ $-0.48, P=.001)$, T1 LV $(r=-0.44, P=.002)$, GM without thalami average MD $(r=-0.36, P=.01)$, NAWM average $\operatorname{MD}(r=-0.37, P=.01)$, and NAWM average FA $(r=0.42$, $P=.005)$; thalamic MD versus the number of thalamic T2 visible lesions $(r=0.41, P=.004)$, T2 LV $(r=0.75, P<$ $.0001)$, T1 LV $(r=0.73, P<.0001)$, GM without thalami average $\mathrm{MD}(r=0.38, P=.01)$, NAWM average MD $(r=$ $0.53, P<.0001)$, and NAWM average FA $(r=-0.40, P=$ $.01)$; and thalamic FA versus number of thalamic T2 visible lesions $(r=-0.31, P=.03)$, T2 LV $(r=-0.59, P<.0001)$, T1 LV $(r=-0.47, P=.001)$, NAWM average MD $(r=-0.41$, $P=.007)$, and NAWM average FA $(r=0.35, P=.02)$.

Table 3 shows the results of the univariate logistic regression analysis of MR imaging - derived quantities of the thalamus and GM without the thalami. Baseline MD of GM without the thalami and thalamic FA change entered the multivariate analysis, together with the variables that were found to be significant at the previous analysis (except GM average MD, which was replaced by GM without the thalami and thalamic average MD). The results of the univariate analysis did not change after masking thalamic T2 visible lesions from thalamic FA and MD maps (data not shown). The final multivariate model included NAWM average MD (OR = 1.46; 95\% CI, 1.12-1.89; $P=.005)$ and FA thalamic change $(\mathrm{OR}=0.84 ; 95 \% \mathrm{CI}, 0.72-0.97 ; P=.02)$ as independent predictors of subsequent EDSS deterioration (Nagelkerke $R^{2}$ $=0.55)$.

\section{Discussion}

In this study, we explored the contribution of thalamic damage to the worsening of disability over a 5 -year period in a relatively large group of patients with PPMS. To this end, we re-analyzed the data collected from a cohort of patients with PPMS studied prospectively at our unit by using conventional imaging and DTI. ${ }^{6,11}$ The use of such a dataset also gave us the unique opportunity to compare the predictive value of thalamic GM damage and its change during a 15-month follow-up period (in terms of lesions, microscopic abnormalities measured by using DTI, and atrophy) on disability accumulation at 5 years with those of other quantities that were identified as predictors of long-term disability accrual at a previous analysis. ${ }^{11}$ Our working hypothesis was that assessing damage to the thalamus should convey important prognostic information due to its strategic location in the brain, its mixed-composition of GM and WM (which represents approximately 5\% of its content ${ }^{13}$ ), and its functional role in motor and cognitive processes.

Our analysis showed that at baseline, patients with PPMS experience significant atrophy as well as DTI microstructural abnormalities of the thalamus in comparison with healthy controls and that such thalamic abnormalities worsen during a relatively short time. Previous studies in patients with early PPMS (within 5 years of symptom onset) have reported that thalamic atrophy ${ }^{9,23,30}$ and thalamic microscopic damage, quantified by using magnetization transfer imaging ${ }^{30}$ and DTI, ${ }^{9}$ are early features of PPMS. However, little is known about the temporal pattern of the evolution of these abnormalities and their relationship with disability accumulation. A 1-year whole-brain voxel-based morphometry longitudinal study ${ }^{23}$ showed progression of thalamic atrophy in 31 patients with early PPMS; however, this was not found to be related to disability worsening.

In addition to atrophy assessment, we also evaluated longitudinal changes of DTI quantities in these patients. Such an analysis showed a progressive increase of thalamic MD and a progressive decrease of thalamic FA. Remarkably, only this latter change was significantly different between patients and controls. Only a few cross-sectional studies used DTI technology to quantify thalamic abnormalities in patients with MS. Similar to our findings, an increased thalamic MD in patients with MS with different clinical disease phenotypes in comparison with healthy controls was found in these studies, on average. ${ }^{9,16,17,31}$ However, contrary to our results, an increased thalamic FA has been described. ${ }^{17,31}$ Several factors might contribute to explaining the discrepancy between ours and previous findings concerning baseline thalamic FA abnormalities, including the fact that previous studies recruited patients 
Table 3: Univariate logistic regression analysis of the predictive value of thalamic volumetry and DTI findings from the GM with and without the thalamus for patients with EDSS scores worsening during 5 years (dependent variable) a $^{\mathrm{a}}$

\begin{tabular}{lccc}
\hline Independent Variables & Units & OR (95\% Cl) & $P$ Values \\
\hline Baseline NTV & $1 \mathrm{~mL}$ & $1.25(0.63-2.45)$ & .53 \\
Baseline thalamic FA & 0.01 & $0.99(0.97-1.02)$ & $1.02(0.98-1.06)$ \\
Baseline thalamic MD & $0.01 \mathrm{~mm}^{2} / \mathrm{s} \times 10^{-3}$ & $1.00(0.91-1.11)$ & .57 \\
Average NTV thalamic volume change & $1 \%$ & $0.87(0.77-0.97)$ & .40 \\
Average thalamic FA change & $1 \%$ & $1.04(0.97-1.11)$ & .94 \\
Average thalamic MD change & $1 \%$ & $1.06(0.66-1.68)$ & .01 \\
Thalamic lesions & n.a. & $2.23(0.40-12.5)$ & .27 \\
Change in thalamic lesions & Yes vs no & $1.02(0.99-1.06)$ & .82 \\
Baseline GM without the thalami FA & 0.01 & $1.14(1.02-1.27)$ & .36 \\
Baseline GM without the thalami MD & $0.01 \mathrm{~mm}^{2} / \mathrm{s} \times 10^{-3}$ & $0.99(0.93-1.05)$ & .22 \\
Average GM without the thalami FA change & $1 \%$ & $1.03(0.92-1.15)$ & .019 \\
Average GM without the thalami MD change & $1 \%$ & .63 \\
\hline
\end{tabular}

a See the text for statistical analysis.

with different MS phenotypes, predominantly relapsing-onset MS, whereas we enrolled patients with PPMS only. In addition, compared with the authors of previous studies, we applied a different strategy to sample the thalamus because we included the entire thalamus and used a GM thalamic mask, whereas other authors used relatively small regions of interest to select a small portion of the thalamus. Remarkably, a reduction of FA values in the thalamus compared with healthy controls has been reported in patients with other neurologic conditions, including Alzheimer disease ${ }^{32}$ and amyotrophic lateral sclerosis, ${ }^{33}$ which likely reflects progressive tissue disorganization and neuronal loss.

To shed light on the possible mechanisms related to thalamic damage, we also investigated the relationship between MR imaging measures of thalamic damage and those of overall and focal disease burden. This analysis showed that thalamic volumetry and DTI abnormalities were more pronounced in patients with PPMS versus those without thalamic lesions. In addition, in the entire sample of patients with PPMS, both thalamic volumetry and DTI values were correlated with the extent of $\mathrm{T} 2$ and $\mathrm{T} 1$ visible lesions of the entire brain as well as with the severity of microscopic damage to the NAWM and the remaining GM. These findings suggest that both WM and GM local inflammatory demyelination as well as changes secondary to axonal transection of fibers passing through areas of diseased brain WM can account for thalamic abnormalities in patients with PPMS.

The univariate analysis of the MR imaging variables evaluated in the present study identified average MD values in the whole GM without the thalami and average thalamic FA changes over the 15-month follow-up as independent predictors of disability worsening after 5 years in our patient cohort. Remarkably, when these 2 variables were combined in a multivariate analysis with those identified at a previous one (ie, baseline EDSS, T2 LV, average lesion MD, average NAWM $\mathrm{MD}$ and $\mathrm{FA}$, and the number of new T2 and T1 lesions at follow-up) ${ }_{11}^{11}$ average GM without the thalami MD did not survive, whereas thalamic FA changes with baseline NAWM average $\mathrm{MD}$ were retained as significantly independent predicting factors. This model explained $55 \%$ of the observed variance of EDSS changes at follow-up. These results support the notion that the assessment of the involvement of critical central nervous system structures, such as the thalamus, might help to explain the progression of disability in patients with
PPMS more than providing an estimate of global GM damage. What is noteworthy, our findings also suggest that the contribution of diffuse NAWM involvement to the long-term accumulation of disability in these patients is not negligible. The role of NAWM and GM involvement in determining overall clinical disability and impairment of selected clinical domains in patients with PPMS is in agreement with the results of recent investigations performed by using different methods of analysis. ${ }^{34,35}$ Remarkably, our results are also in line with a postmortem study ${ }^{36}$ showing that diffuse NAWM injury and GM demyelination are characteristic hallmarks of PPMS.

\section{Conclusions}

Our study demonstrates that in patients with PPMS, the shortterm accrual of damage in the thalamus and the severity of NAWM involvement play a role in determining the accumulation of disability during a medium time period. Further longitudinal studies are warranted to investigate the importance of other deep GM structures and to evaluate the dynamics of disability accrual over longer periods.

\section{References}

1. Thompson AJ, Montalban X, Barkhof F, et al. Diagnostic criteria for primary progressive multiple sclerosis: a position paper. Ann Neurol 2000;47:831-35

2. Miller DH, Leary SM. Primary-progressive multiple sclerosis. Lancet Neurol 2007;6:903-12

3. Rovaris M, Bozzali M, Santuccio G, et al. In vivo assessment of the brain and cervical cord pathology of patients with primary progressive multiple sclerosis. Brain 2001;124:2540-49

4. Rovaris M, Bozzali M, Iannucci G, et al. Assessment of normal-appearing white and gray matter in patients with primary progressive multiple sclerosis: a diffusion-tensor magnetic resonance imaging study. Arch Neurol 2002;59: 1406-12

5. Rovaris M, Gallo A, Falini A, et al. Axonal injury and overall tissue loss are not related in primary progressive multiple sclerosis. Arch Neurol 2005;62:898902

6. Rovaris M, Gallo A, Valsasina P, et al. Short-term accrual of gray matter pathology in patients with progressive multiple sclerosis: an in vivo study using diffusion tensor MRI. Neuroimage 2005;24:1139-46

7. Sastre-Garriga J, Ingle GT, Chard DT, et al. Grey and white matter volume changes in early primary progressive multiple sclerosis: a longitudinal study. Brain 2005;128:1454-60

8. Manfredonia F, Ciccarelli O, Khaleeli Z, et al. Normal-appearing brain T1 relaxation time predicts disability in early primary progressive multiple sclerosis. Arch Neurol 2007;64:411-15

9. Ceccarelli A, Rocca MA, Valsasina P, et al. A multiparametric evaluation of regional brain damage in patients with primary progressive multiple sclerosis. Hum Brain Mapp 2009;30:3009-19

10. Calabrese M, Rocca MA, Atzori M, et al. Cortical lesions in primary progressive multiple sclerosis: a 2-year longitudinal MR study. Neurology 2009;72: $1330-36$ 
11. Rovaris M, Judica E, Gallo A, et al. Grey matter damage predicts the evolution of primary progressive multiple sclerosis at 5 years. Brain 2006;129:2628-34

12. Khaleeli $\mathrm{Z}$, Ciccarelli $\mathrm{O}$, Manfredonia $\mathrm{F}$, et al. Predicting progression in primary progressive multiple sclerosis: a 10-year multicenter study. Ann Neurol 2008;63:790-93

13. Cifelli A, Arridge M, Jezzard P, et al. Thalamic neurodegeneration in multiple sclerosis. Ann Neurol 2002;52:650-53

14. Vercellino M, Masera S, Lorenzatti M, et al. Demyelination, inflammation, and neurodegeneration in multiple sclerosis deep gray matter. J Neuropathol Exp Neurol 2009;68:489-502

15. Ormerod IE, Miller DH, McDonald WI, et al. The role of NMR imaging in the assessment of multiple sclerosis and isolated neurological lesions: a quantitative study. Brain 1987;110(pt 6):1579-616

16. Fabiano AJ, Sharma J, Weinstock-Guttman B, et al. Thalamic involvement in multiple sclerosis: a diffusion-weighted magnetic resonance imaging study. J Neuroimaging 2003;13:307-14

17. Ciccarelli O, Werring DJ, Wheeler-Kingshott CA, et al. Investigation of MS normal-appearing brain using diffusion tensor MRI with clinical correlations. Neurology 2001;56:926-33

18. Wylezinska M, Cifelli A, Jezzard P, et al. Thalamic neurodegeneration in relapsing-remitting multiple sclerosis. Neurology 2003;60:1949-54

19. Inglese M, Liu S, Babb JS, et al. Three-dimensional proton spectroscopy of deep gray matter nuclei in relapsing-remitting MS. Neurology 2004;63:170-72

20. Henry RG, Shieh M, Okuda DT, et al. Regional grey matter atrophy in clinically isolated syndromes at presentation. J Neurol Neurosurg Psychiatry 2008;79: $1236-44$

21. Mesaros S, Rocca MA, Absinta M, et al. Evidence of thalamic gray matter loss in pediatric multiple sclerosis. Neurology 2008;70:1107-12

22. Audoin B, Davies GR, Finisku L, et al. Localization of grey matter atrophy in early RRMS: a longitudinal study. J Neurol 2006;253:1495-501

23. Sepulcre J, Sastre-Garriga J, Cercignani M, et al. Regional gray matter atrophy in early primary progressive multiple sclerosis: a voxel-based morphometry study. Arch Neurol 2006;63:1175-80

24. Kurtzke JF. Rating neurologic impairment in multiple sclerosis: an expanded disability status scale (EDSS). Neurology 1983;33:1444-52
25. Miller DH, Barkhof F, Berry I, et al. Magnetic resonance imaging in monitoring the treatment of multiple sclerosis: concerted action guidelines. J Neurol Neurosurg Psychiatry 1991;54:683-88

26. Rovaris M, Filippi M, Calori G, et al. Intra-observer reproducibility in measuring new putative MR markers of demyelination and axonal loss in multiple sclerosis: a comparison with conventional T2-weighted images. J Neurol 1997; 244:266-70

27. Smith SM, Zhang Y, Jenkinson M, et al. Accurate, robust, and automated longitudinal and cross-sectional brain change analysis. Neuroimage 2002;17: 479-89

28. Smith SM, De Stefano N, Jenkinson M, et al. Normalized accurate measurement of longitudinal brain change. J Comput Assist Tomogr 2001;25:466-75

29. Rocca MA, Mesaros S, Pagani E, et al. Thalamic damage and long-term progression of disability in multiple sclerosis. Radiology 2010;257:463-69

30. Khaleeli Z, Cercignani M, Audoin B, et al. Localized grey matter damage in early primary progressive multiple sclerosis contributes to disability. Neuroimage 2007;37:253-61

31. Tovar-Moll F, Evangelou IE, Chiu AW, et al. Thalamic involvement and its impact on clinical disability in patients with multiple sclerosis: a diffusion tensor imaging study at 3T. AJNR Am J Neuroradiol 2009;30:1380-86

32. Rose SE, Janke AL, Chalk JB. Gray and white matter changes in Alzheimer's disease: a diffusion tensor imaging study. J Magn Reson Imaging 2008;27:20-26

33. Sach M, Winkler G, Glauche V, et al. Diffusion tensor MRI of early upper motor neuron involvement in amyotrophic lateral sclerosis. Brain 2004;127: $340-50$

34. Bodini B, Khaleeli Z, Cercignani M, et al. Exploring the relationship between white matter and gray matter damage in early primary progressive multiple sclerosis: an in vivo study with TBSS and VBM. Hum Brain Mapp 2009;30: 2852-61

35. Penny S, Khaleeli Z, Cipolotti L, et al. Early imaging predicts later cognitive impairment in primary progressive multiple sclerosis. Neurology 2010;74: 545-52

36. Kutzelnigg A, Lucchinetti CF, Stadelmann C, et al. Cortical demyelination and diffuse white matter injury in multiple sclerosis. Brain 2005;128:2705-12 